\title{
Yellow Nail Syndrome Successfully Treated with Oral Terbinafine and Topical Minoxidil
}

This article was published in the following Dove Press journal:

Clinical, Cosmetic and Investigational Dermatology

\section{Maysoon Algain}

Department of Dermatology, King Abdulaziz University, Jeddah, Saudi Arabia

\begin{abstract}
Yellow nail syndrome (YNS) is a rare disease of unknown etiology that is characterized by varying degrees of pulmonary manifestations, lymphedema, and yellow discoloration of the nails. Herein, we report the efficacy of oral terbinafine and topical minoxidil in treating the associated nail abnormalities of YNS in a 66-year-old woman. The patient presented with yellow, brittle, slow-growing nails, which had developed progressively over the past 8 months; left ankle edema; a chronic, purulent, productive cough; and exertional dyspnea. Based on these symptoms, she was diagnosed with YNS. She failed to respond to treatment with fluconazole and vitamin E; however, she was successfully treated with oral terbinafine and topical minoxidil. The treatment was well tolerated, and we speculate that its mode of action includes promoting lymphatic formation and barrier enhancement, thereby improving the distal lymphedema, in addition to distal vasodilation that accelerates the nail growth. To our knowledge, this is the first report of successful treatment of YNS using these agents.
\end{abstract}

Keywords: exertional dyspnea, lymphedema, productive cough, case report

\section{Introduction}

Yellow nail syndrome (YNS) is rare, has an unknown etiology, and is characterized by varying degrees of pulmonary manifestations, lymphedema, and yellow discoloration of the nails. The mechanisms underlying this syndrome remain poorly understood; however, dysfunctional lymphatics are speculated to play a role. Here, we report the efficacy of oral terbinafine and local minoxidil in treating the associated nail abnormalities in a 66-year-old woman diagnosed with YNS.

\section{Case Report}

A 66-year-old woman with no medical or family history sought advice for her yellow, brittle, slow-growing nails, a condition which had developed progressively over the past 8 months, together with edema of the left ankle; a chronic, purulent, productive cough; nasal congestion; and exertional dyspnea. She had never smoked and denied any occupational exposure to potential risk factors. The physical examination found thickening and yellowish discoloration of all nails with onycholysis, onychoschizia, and some degree of subungual hyperkeratosis (Figure $1 \mathrm{~A}-\mathrm{C}$ ). Lung auscultation revealed unilateral coarse crackles. Computed tomography demonstrated right lower lobe bronchiectasis and left maxillary sinusitis. Bronchial endoscopy found purulent secretions, while the biopsy indicated non-specific inflammatory changes. Streptococcus pneumoniae was isolated from the sputum samples. Her pulmonary
Correspondence: Maysoon Algain

Department of Dermatology,

King Abdulaziz University, Jeddah, Saudi

Arabia

Tel +96650300030l

Email maysoonalgain@gmail.com 


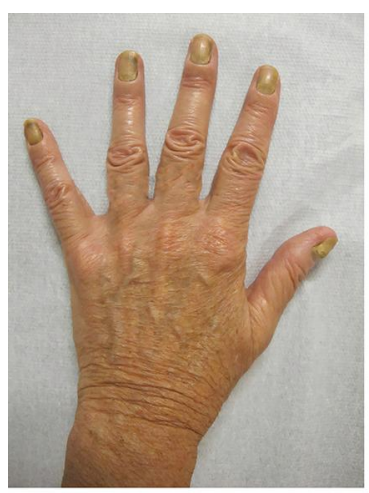

A

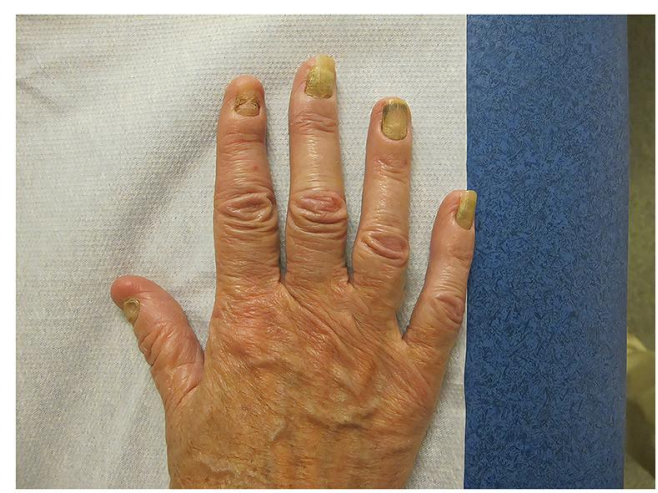

B

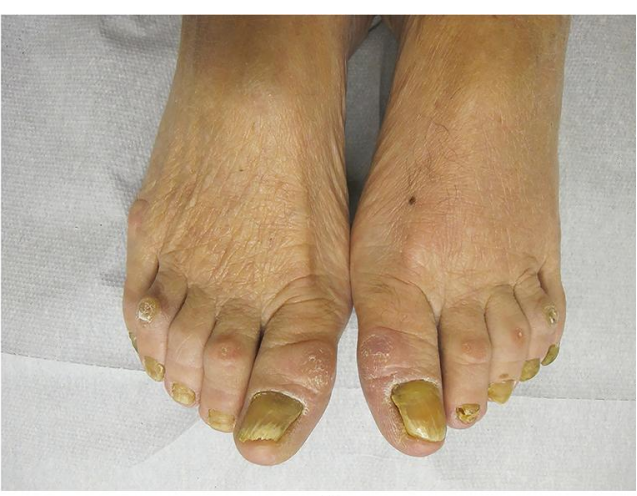

C

Figure I Thickening and yellow discoloration of all nails with onycholysis, onychoschizia, and some degree of subungual hyperkeratosis (A-C).

function tests were normal, and routine biological tests, abdominal and pelvic ultrasound examinations were unremarkable. Potassium hydroxide preparation test and mycological cultures of the fingernails were negative. Accordingly, the patient was diagnosed with YNS. The respiratory symptoms improved through treatment with Augmentin ${ }^{\circledR}$ and physiotherapy. However, episodes of pneumonia regularly occurred during the follow-up period.

Since yellow nails have been reported to improve with fluconazole $300 \mathrm{mg}$ per week and vitamin E $1000 \mathrm{mg}$ per day, ${ }^{1}$ the patient was treated continually with this regimen for 7 months. However, no changes in the nail appearance or growth were observed. Therefore, fluconazole and vitamin E were discontinued and terbinafine $250 \mathrm{mg}$ per day was started. Two months later, clarithromycin $250 \mathrm{mg}, 3$ times per week, was added to control the respiratory tract infections, and the frequency of these episodes clearly decreased. Five months after starting the terbinafine treatment, all toenails and 6 fingernails showed major improvements, and the

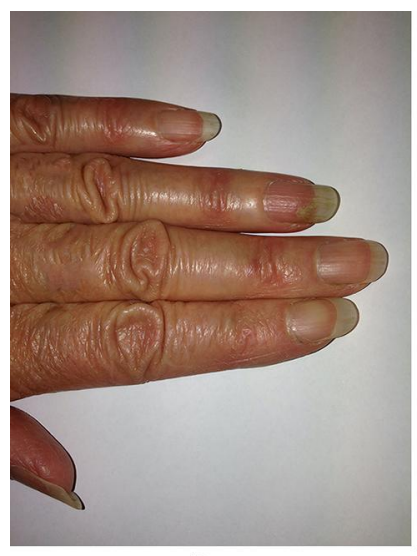

A

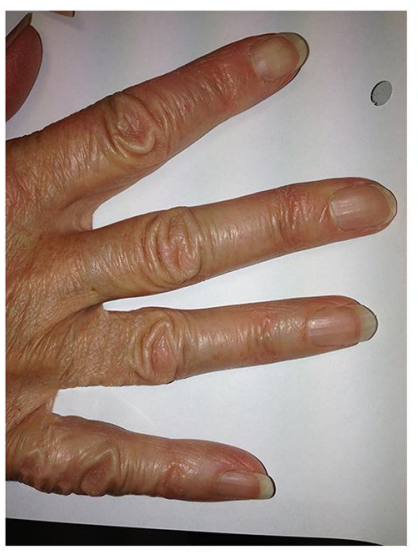

B ankle edema disappeared. The degree of improvement was $30-80 \%$. Topical minoxidil $2 \%$ was additionally prescribed to be applied around the 4 non-responding nails while continuing the terbinafine treatment. After 6 months, the yellow appearance persisted only on the distal half of the nails on the 2 big toes, and discrete onycholysis was noted on the fourth right-hand nail. Subsequently, the terbinafine dose was tapered off every other day for 6 months, and complete remission was observed in all nails, except for those on the big toes (Figure 2A-C). During 72 months of follow-up, there was no recurrence of the patient's nail lesions. During the treatment, monthly monitoring of complete blood count and liver function tests was done, the results of which were normal, and the patient had no side effects. The patient provided written informed consent.

\section{Discussion}

YNS is a rare disease with an unknown pathogenesis, although lymphatic dysfunction has been reported to be

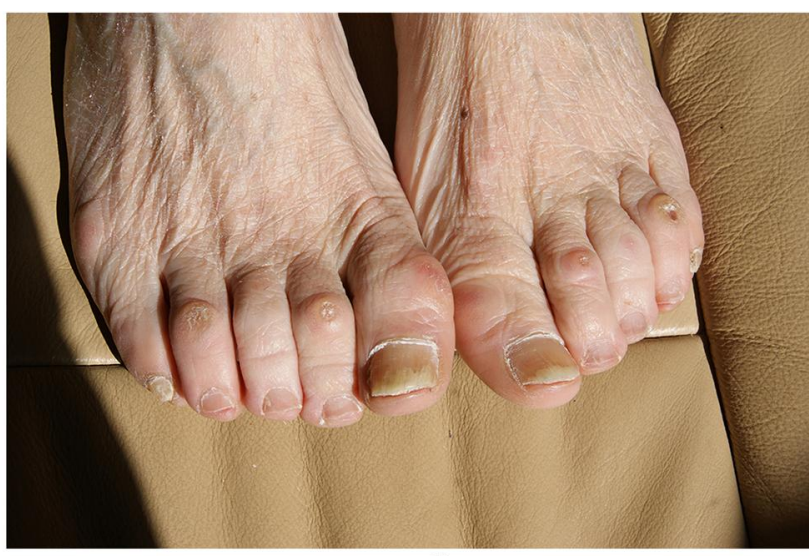

C

Figure 2 After treatment with oral terbinafine and topical minoxidil, complete remission in all the nails was achieved except on the big toes (A-C). 
the predominant mechanism underlying the clinical manifestations. In a case series of 13 patients with YNS, 8 had peripheral edema. Of these 8 , a further 4 showed lymphatic abnormalities on lymphography, including a decrease in the size and number of lymphatic and hypoplastic vessels and a decrease in lymphatic extravasation, with the abnormalities differing in all cases. ${ }^{1}$ Moreover, abnormal lymphatics were detected in the non-edematous limbs. The fact that nail changes and lymphatic anomalies may become apparent simultaneously, often for the first time late in life, suggests that they are likely related. ${ }^{2}$ Accordingly, YNS manifestations such as nail changes, respiratory signs, and primary lymphedema were all present in our patient.

The treatment of YNS is challenging. Treatment may be indicated for the psychosocial implications of having disfigured nails as well as for impaired fine finger and toe function. However, there is currently no standard treatment for nail involvement in this syndrome. Different modalities targeting nail growth have been reported, including topical or oral vitamin E, triamcinolone injections, and oral zinc. ${ }^{3}$ Recently, the results of prolonged fluconazole and itraconazole use have been reported, with varying outcomes. In the above-mentioned series of 13 patients, fluconazole and alpha-tocopherol treatment resulted in the curing and improvement of the nail symptoms in 8 and 2 patients, respectively, ${ }^{1}$ whereas in another retrospective study of 9 patients who received vitamin $\mathrm{E}$ and itraconazole, only 1 patient showed complete recovery, and the other 3 showed slight improvement. ${ }^{4}$

Our patient failed to respond to fluconazole but was nearly cured using terbinafine and topical minoxidil. We cannot exclude the role of clarithromycin; however, in our literature review, we only identified 6 patients reported to have YNS treated by clarithromycin, with the dose ranging from 200-600 mg/day. Patients who received clarithromycin $200 \mathrm{mg} /$ day $(\mathrm{n}=4)$ showed no improvement in their nail symptoms; however, when the dose was increased to 400-600 mg/day, all patients had partial to complete recovery of their yellow nails. ${ }^{5,6}$ This dose was 4-6 times higher than that administered to our patient. ${ }^{5}$ To date, there have been no reports on the treatment of YNS with terbinafine. In 2 studies on onychomycosis, nail growth was found to be $30 \%$ faster in terbinafine-treated patients compared with griseofulvin-treated patients. ${ }^{7,8}$ This may be due to terbinafine's effect on nail growth independent from its antifungal activity. In addition, the cultures did not yield any causative pathogen in our patient; however, she showed improvement in nail growth, thus supporting the former statement. The mechanism of action of terbinafine may involve interference with the regulation of the rho-kinase-mediated pathway, specifically RhoC, thereby improving distal lymphedema. ${ }^{9}$ At the molecular level, terbinafine increases membranebound RhoC levels and decreases phosphorylated focal adhesion kinase (FAK) levels. ${ }^{9}$ In turn, RhoC activation leads to up-regulation of vascular endothelial growth factor- $\mathrm{C}$, thereby promoting lymphangiogenesis. ${ }^{10}$ In addition, the loss of FAK in the endothelial cells enhances cell attachment, leading to the formation of a tighter endothelial monolayer, which consequently enhances the lymphatic barrier function. ${ }^{11}$

Terbinafine acts as a squalene epoxidase enzyme inhibitor that prevents the breakdown of squalene, leading to intracellular squalene accumulation. ${ }^{12}$ Squalene is a triterpene that carries oxygen directly to cell membranes independent of hemoglobin. Moreover, squalene functions as a scavenger for reactive oxygen species (ROS); therefore, it lowers the intracellular level of ROS and increases the bioavailability of nitric oxide, which is a strong vasodilator mediator. $^{13,14}$ As a result, intracellular squalene increases the blood flow and improves the oxygen supply to the nail, leading to accelerated nail growth.

Moreover, the use of topical minoxidil for treating nonresponsive fingernails allowed further improvement in our patient, likely through vasodilatation. In a pilot study, 32 healthy participants applied topical minoxidil on the ring, index, and middle fingernails, resulting in accelerated nail growth. They excluded the thumb and the little finger nails owing to their known tendency to have slower growth. ${ }^{15}$ Our patient showed complete resolution of yellow nails except for the distal part of the big toes; this could be related to the physiological tendency of the big toenails to have the slowest growth rate, especially in elderly women. ${ }^{16}$

In summary, we reported the first case of a patient with YNS who failed to respond to fluconazole and vitamin E but showed near-complete recovery using terbinafine and topical minoxidil. The treatment was well tolerated, and we speculate that its mode of action includes vasodilation and promoting lymphatic formation and barrier enhancement, thereby improving distal lymphedema and blood supply to the nails.

\section{Data Sharing Statement}

The datasets supporting the conclusions of this article are included in the article. 


\section{Ethics Statement}

Institutional approval was not required to publish the case details.

\section{Consent to Publish}

Written informed consent was obtained from the patient, and she agreed to have the case details and any accompanying images published.

\section{Funding}

No sources of funding or support to be declared.

\section{Disclosure}

The author reports no conflicts of interest in this work.

\section{References}

1. Baran R, Thomas L. Combination of fluconazole and alpha-tocopherol in the treatment of yellow nail syndrome. $J$ Drugs Dermatol. 2009;8:276-278.

2. Samman PD, White WF. The 'yellow nail' syndrome. Br J Dermatol. 1964;76:153-157. doi:10.1111/j.1365-2133.1964.tb14499.x

3. Al Hawsawi K, Pope E. Yellow nail syndrome. Pediatr Dermatol. 2010;27:675-676. doi:10.1111/j.1525-1470.2010.01338.x

4. Piraccini BM, Urciuoli B, Starace M, Tosti A, Balestri R. Yellow nail syndrome: clinical experience in a series of 21 patients. $J$ Dtsch Dermatol Ges. 2014;12:131-137. doi:10.1111/ddg.12216

5. Matsubayashi S, Suzuki M, Suzuki T, et al. Effectiveness of clarithromycin in patients with yellow nail syndrome. BMC Pulm Med. 2018;18:138. doi:10.1186/s12890-018-0707-4

6. Suzuki M, Yoshizawa A, Sugiyama H, et al. A case of yellow nail syndrome with dramatically improved nail discoloration by oral clarithromycin. Case Rep Dermatol. 2011;251-258. doi:10.1159/ 000334734
7. Faergemann J, Anderson C, Hersle K, et al. Double-blind parallel-group comparison of terbinafine and griseofulvin in the treatment of toenail onychomycosis. $J$ Am Acad Dermatol. 1995;32:750-753. doi:10.1016/0190-9622(95)91454-4

8. Baran R, Belaich S, Beylot $\mathrm{C}$, et al. Comparative multicentre double-blind study of terbinafine (250 mg per day) versus griseofulvin ( $1 \mathrm{~g}$ per day) in the treatment of dermatophyte onychomycosis. J Dermatolog Treat. 1997;8:93-97. doi:10.3109/09546639709160278

9. Ho PY, Zhong WB, Ho YS, Lee WS. Terbinafine inhibits endothelial cell migration through suppression of the rho-mediated pathway. Mol Cancer Ther. 2006;5:3130-3138. doi:10.1158/1535-7163.MCT-060457

10. Wu M, Wu ZF, Kumar-Sinha C, Chinnaiyan A, Merajver SD. RhoC induces differential expression of genes involved in invasion and metastasis in MCF10A breast cells. Breast Cancer Res Treat. 2004;84:3-12. doi:10.1023/B:BREA.0000018426.76893.21

11. Arnold KM, Goeckeler ZM, Wysolmerski RB. Loss of focal adhesion kinase enhances endothelial barrier function and increases focal adhesions. Microcirculation. 2013;20:637-649. doi:10.1111/ micc. 12063

12. Ryder NS. Terbinafine: mode of action and properties of the squalene epoxidase inhibition. $\mathrm{Br} J$ Dermatol. 1992;126(Suppl 39):2-7. doi:10.1111/j.1365-2133.1992.tb00001.x

13. Ibrahim N, Fairus S, Zulfarina MS, Naina Mohamed I. The efficacy of squalene in cardiovascular disease risk-a systematic review. Nutrients. 2020;12:414. doi:10.3390/nu12020414

14. Sriram K, Laughlin JG, Rangamani P, et al. Shear-induced nitric oxide production by endothelial cells. Biophys J. 2016;111:208-221. doi:10.1016/j.bpj.2016.05.034

15. Aiempanakit K, Geater A, Limtong P, et al. The use of topical minoxidil to accelerate nail growth: a pilot study. $J$ Invest Dermatol. 2017;56:788-791. doi:10.1111/ijd.13620

16. Orentreich N, Markofsky J, Vogelman JH. The effect of aging on the rate of linear nail growth. J Invest Dermatol. 1979;73:126-130. doi:10.1111/1523-1747.ep12532799

\section{Publish your work in this journal}

Clinical, Cosmetic and Investigational Dermatology is an international, peer-reviewed, open access, online journal that focuses on the latest clinical and experimental research in all aspects of skin disease and cosmetic interventions. This journal is indexed on CAS.
The manuscript management system is completely online and includes a very quick and fair peer-review system, which is all easy to use. Visit http://www.dovepress.com/testimonials.php to read real quotes from published authors. 